\title{
Influencia de la variabilidad ambiental de la Laguna la Cruz (Sonora) en el crecimiento y condición del ostión del Pacífico Crassostrea gigas
}

\author{
Influence of environmental variability of La Cruz Lagoon (Sonora) on growth and condition of the Pacific \\ oyster Crassostrea gigas
}

\begin{abstract}
Felipe de Jesús Reynaga-Franco', Jorge Chávez-Villalba², Ramón Héctor Barraza-Guardado', Edgar Alcántara-Razo², José Alfredo Arreola-Lizárraga² ${ }^{2}$ Reina Castro-Longoria ${ }^{1}$ y José Manuel Grijalva-Chon ${ }^{1 *}$

Universidad de Sonora, Departamento de Investigaciones Científicas y Tecnológicas, Av. Luis D. Colosio s/n, Hermosillo, Sonora 83000, México.

2 Centro de Investigaciones Biológicas del Noroeste (CIBNOR), Unidad Sonora, Apdo. Postal 349, Guaymas, Sonora 85454, México.
\end{abstract}

\section{RESUMEN}

Se estudió el efecto de la temperatura, salinidad, oxígeno, clorofila a y seston sobre el crecimiento y condición de ostiones Crassostrea gigas cultivados en la laguna La Cruz (Sonora, México). Se realizaron biometrías (altura, longitud y peso) quincenales (abril-agosto) y mensuales (septiembrediciembre) de los ostiones y se utilizó la inferencia multimodelo para describir el crecimiento. Se determinó por análisis de PCR que los patógenos (OsHV-1, Marteilia refringens y Perkinsus marinus) estuvieron ausentes en semillas y ostiones adultos. La temperatura influenció el crecimiento y la condición cuando se presentaron los valores máximos en verano. En este periodo los ostiones detienen su crecimiento en concha pero el alimento disponible, indicado por la clorofila y seston, permite la formación de carne. Esta estrategia parece una adaptación de los ostiones a la variación de temperatura y sitúa su desarrollo óptimo entre $20-30{ }^{\circ} \mathrm{C}$. En estas condiciones, el modelo que mejor describió el crecimiento fue el Schnute caso 1. Estos resultados demuestran que a pesar de temperaturas extremas los ostiones tuvieron un buen desarrollo durante su cultivo.

Palabras clave: Crassostrea gigas, ostión del Pacífico, acuacultura, modelo de Schnute, Golfo de California.

\section{ABSTRACT}

It was studied the effect of temperature, salinity, oxygen, chlorophyll $a$ and seston on the growth and condition of Crassostrea gigas oysters cultivated in La Cruz lagoon (Sonora, Mexico). Biometrics (height, length and weight) of oysters were carried out fortnightly (April-August) and monthly (September-December), and multimode inference was used to describe the growth. It was determined by PCR analysis that pathogens (OsHV-1, Marteilia refringens and Perkinsus marinus) were absent in spat and adult oysters. The temperature influenced the growth and condition when the maximum values were detected in summer. In this period the oysters stop their growth in shell but the available food, indicated by the chlorophyll and seston, allowed the formation of meat. This strategy seems an adaptation of the oysters to the variation of temperature and situates its optimal

Volumen XXI, Número 2 development between $20-30^{\circ} \mathrm{C}$. Under these conditions, the model that best described the growth was the Schnute case 1. These results show that in spite of extreme temperatures the oysters showed a good development during cultivation.

Keywords: Crassostrea gigas, Pacific oyster, aquaculture, Schnute model, Gulf of California.

\section{INTRODUCCIÓN}

Los moluscos son el tercer grupo de interés acuícola en el mundo, solamente superados por los cultivos de peces y de crustáceos; dentro de los moluscos destacan los bivalvos y en particular el ostión del Pacífico Crassostrea gigas (Thunberg, 1793), también conocido como ostión japonés, la cual es la ostra más cultivada en el mundo (FAO, 2018). La especie se distingue por un rápido crecimiento, una amplia tolerancia a diversas condiciones ambientales y buena resistencia contra patógenos, esto ha propiciado que la especie se cultive prácticamente en todos los mares del mundo (Diederich et al., 2005). China es el principal productor mundial de la especie aportando más del $80 \%$ de la producción mundial, le siguen Japón, la República de Corea, Francia y Estados Unidos de América (EUA) (FAO, 2016). En este último país, el cultivo del ostión del Pacífico genera 227 millones de dólares al año y más de 15,000 empleos (FAO, 2018).

En México, la especie se introdujo en San Quintín, Baja California, como una alternativa para diversificar la acuacultura en el país (Rosa-Vélez et al., 1991). Los primeros cultivos se realizaron a partir de semilla importada de los EUA y fueron cultivadas en postas rústicas, pero actualmente existen diversos laboratorios de producción de semilla en el noroeste de México (Chávez-Villalba, 2014). En México la tasa de crecimiento para la especie es una de las más rápidas en el mundo, por ejemplo Castillo-Durán et al. (2010) mostraron que la especie crece $0.221 \mathrm{~mm} /$ día. Se ha reportado que el crecimiento de la especie está relacionado con las variables ambientales, particularmente con la temperatura y la disponibilidad de alimento (Chávez-Villalba et al., 2007).

Las lagunas del Golfo de California presentan una alta variabilidad estacional, atribuida a la escasa precipitación y las altas temperaturas de la región (Arreola-Lizárraga, 2003).

*Autor para correspondencia: José Manuel Grijalva-Chon Correo electrónico: manuel.grijalva@unison.mx 
Algunas de estas lagunas son ecosistemas altamente productivos (Gómez-León et al., 2018), particularmente la laguna La Cruz es considerada como una con la mayor productividad primaria del Golfo de California, pero con marcadas variaciones estacionales (Castro-Longoria et al., 1991; Valdez-Holguín et al., 1994; Gárate-Lizárraga et al., 2013). Aunque se ha evaluado la influencia de las condiciones extremas de temperatura de La Cruz sobre la expresión de genes del ostión del Pacífico (Valenzuela-Castillo et al., 2015), se requiere más información de como esta productividad y variabilidad ambiental influyen en el crecimiento de la especie.

Tradicionalmente el crecimiento es el principal indicador de éxito en los cultivos de los bivalvos y para monitorearlo se emplean mediciones puntuales de la concha (alto, largo y ancho), así como la variación del peso total del organismo en diferentes intervalos de tiempo. No obstante, estas mediciones no permiten identificar patrones de crecimiento, $\mathrm{ni}$ identificar la influencia de las variables ambientales sobre el crecimiento de la especie en los cultivos comerciales. Una alternativa a las mediciones tradicionales es la implementación de la inferencia multimodelo, la cual permite identificar el mejor modelo y toma en cuenta variaciones estacionales para describir los patrones de crecimiento de la especie de interés (Chávez-Villalba y Aragón-Noriega, 2015; GóngoraGómez et al., 2017). Junto con el crecimiento, los índices de condición revelan el estado fisiológico de los organismos cultivados debido a que son afectados por los cambios estacionales de las variables ambientales y por eventos asociados a la reproducción (Gasmi et al., 2017). Los índices brindan además información complementaria de interés a los productores ya que son indicadores de la calidad del producto que será comercializado.

Por otro lado, así como las variables ambientales, los patógenos son un factor limitante en el cultivo del ostión del Pacífico. Por ejemplo, el herpesvirus de los ostreidos tipo 1 (OsHv-1) y sus variantes, así como los protozoarios Marteilia refringens y Perkinsus marinus son los principales patógenos que afectan a la especie pudiendo producir altas mortalidades tanto en los cultivos como en los laboratorios de producción de semilla (Vázquez-Yeomans et al., 2004, 2010; Cáceres-Martínez et al., 2008, 2012; Enríquez-Espinoza et al., 2010, 2015; Villanueva-Fonseca y Escobedo-Bonilla, 2013; Escobedo-Fregoso et al., 2015; Grijalva-Chon et al., 2015; De la Re-Vega et al., 2017; Martínez-García et al., 2017;). Estos patógenos están presentes en el Golfo de California y su proliferación está relacionada con las fluctuaciones de las variables ambientales. Las variaciones, particularmente cuando se llega a valores extremos (por ejemplo de temperatura), favorecen el estrés de los organismos en cultivo disminuyendo su capacidad de respuesta a procesos infecciosos (Fuhrmann et al., 2018). El monitoreo continuo de patógenos es fundamental para tratar de mitigar su impacto en los cultivos comerciales.

Como se puede observar, la variabilidad ambiental de las lagunas influye en gran cantidad de procesos que afectan el desarrollo de los organismos, por lo que es importante documentar su influencia en el crecimiento de especies con interés comercial. Es por ello que el objetivo de este trabajo es analizar la influencia de la variabilidad ambiental de la laguna La Cruz en el crecimiento y la condición del ostión $C$. gigas durante un ciclo de cultivo.

\section{MATERIALES Y MÉTODOS}

Se realizó la compra (siguiendo los protocolos convencionales) de un lote de 10,000 semillas de ostión del Pacífico en un laboratorio proveedor habitual de los ostricultores de la región. El lote se recibió el día 9 de abril del 2014 y se sembró ese mismo día en un sistema tradicional de cultivo en la laguna La Cruz, Sonora ( $28^{\circ} 47^{\prime} 58.0^{\prime \prime} \mathrm{N}, 111^{\circ} 54^{\prime} 55.5^{\prime \prime} \mathrm{W}$, Fig. 1). El experimento se llevó a cabo del 9 de abril al 12 de diciembre de 2014, y durante este periodo se llevaron a cabo muestreos cada 15 días durante los primeros cinco meses y luego mensualmente hasta el final del estudio.

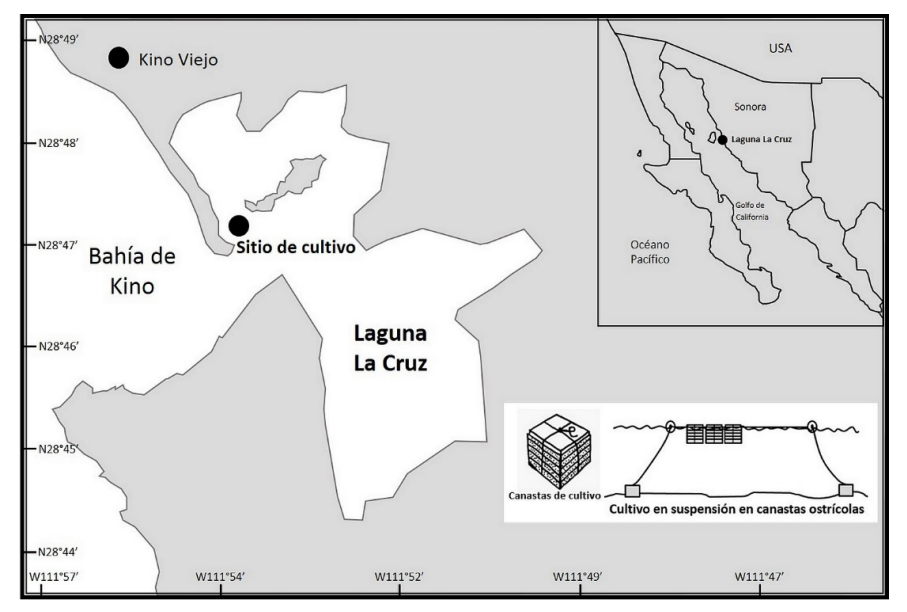

Figura 1. Localización del área de estudio y sistema de cultivo utilizado. Figure 1. Location of the study area and cultivation system used.

\section{Variables ambientales}

La temperatura, la salinidad y el oxígeno disuelto fueron medidos en cada muestreo con una sonda multisensor YSI-85. Al mismo tiempo se colectó una muestra de un litro de agua para estimar la concentración de clorofila $a$ y de seston. La concentración de clorofila se determinó mediante la metodología descrita por Parsons et al. (1984), mientras que la concentración de seston se llevó a cabo siguiendo los procedimientos descritos en Strickland y Parsons (1972).

\section{Crecimiento de los ostiones}

Los ostiones se cultivaron usando dos réplicas en un sistema en suspensión en cajas ostrícolas siguiendo los procedimientos (mantenimiento, limpieza, etc.) llevados a cabo por los productores de la región. En cada muestreo se seleccionaron al azar dos grupos (uno por cada réplica) de 50 organismos cada uno y se transportaron en hielo a la Unidad Experimental Kino de la Universidad de Sonora. A los organismos seleccionados se les revisó el estado de las valvas, después se lavaron con agua destilada y en caso de ser necesario se retiraron epibiontes con una espátula. Para 
determinar el crecimiento se registró la altura y el largo de la concha con un vernier digital, posteriormente cada ostión fue pesado en una balanza de precisión para obtener su peso total.

\section{Índice de condición}

En este trabajo se evaluó el índice de condición considerando que la etapa de semilla corresponde a una altura de concha entre 5 y $10 \mathrm{~mm}$, y la etapa de juvenil y adulto corresponden a alturas mayores de $10 \mathrm{~mm}$. En el caso de las semillas, se utilizó el índice de condición reportado por Barraza-Guardado et al. (2009) descrito por la ecuación:

$$
I C=\frac{M O}{P S} * 100
$$

dónde MO es la materia orgánica y PS es el peso seco. En la etapa de juveniles y adultos el índice de condición fue estimado según lo propuesto por Crosby y Gale (1990) descrito por la ecuación:

$$
I C=\frac{P S T * 1000}{P S V} * 100
$$
de las valvas.

dónde PST es el peso seco total y PSV es el peso seco

\section{Modelación del crecimiento individual}

Se evaluaron tres modelos de crecimiento y se probaron seis ecuaciones, lo anterior utilizando datos de alturaedad para determinar cuál de los modelos representa mejor los datos. Los modelos utilizados fueron los que se describen a continuación. El modelo de crecimiento de von Bertalanffy descrito por la ecuación:

$$
L(t)=L_{\infty}\left(1-e^{-k\left(t-t_{0}\right)}\right)
$$

El modelo logístico descrito por la ecuación:

$$
L(t)=L_{\infty}\left(1+e^{-k 2\left(t-t^{*}\right)}\right)^{-1}
$$

Estos dos modelos utilizan los siguientes parámetros: $L(t)$ es la longitud a la edad $t$; $t$ es la edad en años; $L \infty$ es la longitud promedio de los organismos más viejos (parámetro de longitud asintótico); $k$ determina la rapidez en que $L \infty$ es alcanzada (parámetro de curvatura); $t_{0}$ es la edad hipotética en la cual el organismo tiene longitud cero (parámetro de la condición inicial); $k 2$ es el parámetro de la tasa de crecimiento relativa; $t^{*}$ es el punto de inflexión en la curva sigmoides.

El otro modelo utilizado fue el de crecimiento de Schnute (Schnute, 1981), el cual es un modelo de crecimiento basado en cuatro parámetros que toma cuatro formas matemáticas dependiendo de los valores de $a$ y $b$ en relación a 0 . En este estudio se utilizó el modelo Schnute caso 1 cuando $a$ $\neq 0, b \neq 0$, con la ecuación:

$$
L_{t}=\left\{Y_{1}^{b}+\left(Y_{2}^{b}-Y_{1}^{b}\right)\left[\frac{1-e^{-a\left(t-\tau_{1}\right)}}{1-e^{-a\left(\tau_{2}-\tau_{1}\right)}}\right]\right\}^{\frac{1}{b}}
$$

Se utilizó el caso 2 del modelo Schnute cuando $a \neq 0$, $b=0$, con la ecuación

$$
L_{t}=Y_{1} \exp \left[\ln \left(\frac{Y_{2}}{Y_{1}}\right) \frac{1-e^{-a\left(t-\tau_{1}\right)}}{1-e^{-a\left(\tau_{2}-\tau_{1}\right)}}\right]
$$

El caso 3 del modelo Schnute fue cuando $a=0, b \neq 0$, con la ecuación:

$$
L_{t}=\left\{Y_{1}^{b}+\left(Y_{2}^{b}-Y_{1}^{b}\right)\left[\frac{t-\tau_{1}}{\tau_{2}-\tau_{1}}\right]\right\}^{\frac{1}{b}}
$$

En el caso 4 del modelo Schnute fue cuando $a=0, b=$ 0 , con la ecuación

$$
L_{t}=Y_{1} \exp \left[\ln \left(\frac{Y_{2}}{Y_{1}}\right) \frac{t-\tau_{1}}{\tau_{2}-\tau_{1}}\right]
$$

Estas ecuaciones utilizan los siguientes parámetros: $\tau_{1}$ es la menor edad en el set de datos; $\tau_{2}$ es la mayor edad en el set de datos; $a$ es el parámetro de la tasa de crecimiento relativa; $b$ es la tasa del crecimiento incremental relativo; $Y_{1}$ es la talla a la edad $\tau_{1} ; Y_{2}$ es la talla en la edad $\tau_{2}$

Los modelos de Schnute fueron ajustados usando máxima verosimilitud, cuyo algoritmo de ajuste está basado en la ecuación:

$$
L L(\Phi \mid \text { data })=-\left(\frac{n}{2}\right)(\ln (2 \pi)+2 * \ln (\sigma)+1)
$$

dónde $\Phi$ representa los parámetros del modelo y $\sigma$ representa la desviación estándar del error, el cual fue calculado usando la ecuación:

$$
\sigma=\sqrt{\frac{\sum\left(\ln L_{o b s}-\ln \hat{L}\right)^{2}}{n}}
$$

El enfoque multimodelo se utilizó para determinar el mejor modelo (Katsanevakis, 2006) basado en el criterio AIC (Criterio de Información de Akaike), definido como: $\mathrm{AIC}=-2 \mathrm{LL}$ $+2 \theta_{i}$ donde $L L$ es el máximo log-likelihood y $\theta_{i}$ es el número de parámetros de cada modelo muestreado. Las diferencias en el AIC ( $\Delta_{i}=A I C_{i}-A I C_{\min }$ ), fueron estimadas entre todos los modelos usados en este estudio. El modelo con el menor valor de AIC fue seleccionado como el mejor modelo. Para decidir estadísticamente la aptitud del modelo para describir los datos, se evaluó un criterio propuesto por Burnham y Anderson (2002), en el cual $\Delta \mathrm{i}<2$ es evidencia de apoyo sustancial, $4<\Delta \mathrm{i}<7$ sugiere algo de evidencia y $\Delta \mathrm{i}>10$ no muestra evidencia esencial en el ajuste de los datos. Finalmente, la plausibilidad de cada modelo fue estimada usando la siguiente fórmula para el peso de Akaike: 


$$
W_{i}=\frac{e\left(-0.5 \Delta_{i}\right)}{\sum_{k=1}^{4} e\left(-0.5 \Delta_{k}\right)}
$$

\section{Análisis de patógenos}

Para el análisis de patógenos se tomaron 100 semillas antes de la siembra y 30 ostiones adultos al final del período de cultivo. Todas las muestras se conservaron en etanol $(\geq$ 95\%). En el caso de la semilla, estos se agruparon en lotes de cinco organismos (incluido el caparazón) para obtener un total de 20 lotes. Los procedimientos para detectar los patógenos fueron los mismos para semillas y adultos utilizando métodos reportados en la literatura. Para OsHV-1 se basó en Arzul et al. (2014) y las condiciones de amplificación fueron las descritas por Renault et al. (2000) y Vigneron et al. (2000). Para Perkinsus marinus se usó lo reportado por Casas et al. (2002) y Audemard et al. (2004), con las condiciones de amplificación informadas por Enríquez-Espinoza et al. (2010). Finalmente, para Marteilia refringens se realizó una PCR anidada con oligos reportados por López-Flores et al. (2004).

\section{RESULTADOS Y DISCUSIÓN}

\section{Variables ambientales}

La temperatura, salinidad y oxígeno disuelto en el sitio de estudio presentaron variaciones estacionales durante el cultivo experimental (Fig. 2a). La temperatura promedio fue de $28.4 \pm 4.5^{\circ} \mathrm{C}$, con valores máximos en agosto $\left(33^{\circ} \mathrm{C}\right)$ y mínimos en diciembre $\left(19.1^{\circ} \mathrm{C}\right)$. El promedio de salinidad fue de $37.7 \pm 1.4$ y los valores mostraron un patrón descendente de
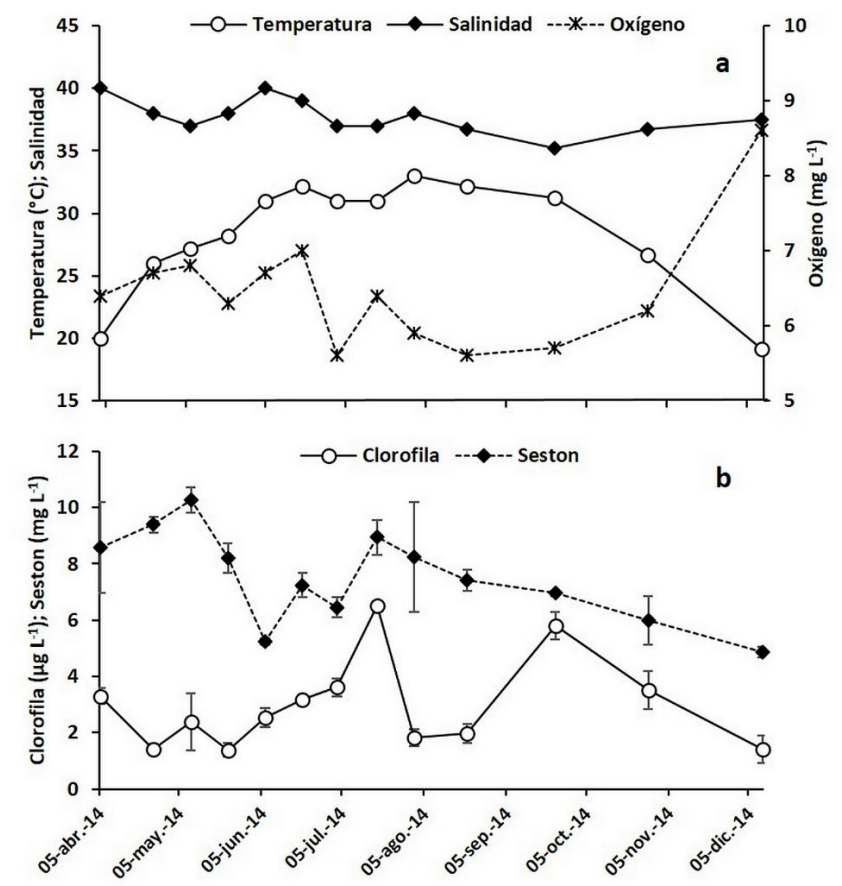

Figura 2. Valores de temperatura, salinidad y oxígeno (a) y de clorofila a y seston (b) registrados en la laguna La Cruz, Sonora.

Figure 2. Values of temperature, salinity and oxygen (a), and chlorophyll $a$ and seston (b) registered at La Cruz lagoon, Sonora. abril (40) a septiembre (35.2), para luego volver a incrementar hasta el final del estudio (37.5). El promedio de oxígeno fue de $6.4 \pm 0.8 \mathrm{mg} \mathrm{L}^{-1}$ con oscilación de sus valores entre 5.5 $-7 \mathrm{mg} \mathrm{L}^{-1}$ y con un valor máximo de $8.6 \mathrm{mg} \mathrm{L}^{-1}$ en diciembre. La temperatura y la concentración de oxígeno presentaron patrones inversos. Los valores mayores de temperatura coincidieron con las menores concentraciones de oxígeno, lo cual es común en las lagunas costeras del Golfo de California (Valdez-Holguín et al., 1994; Páez-Osuna et al., 2016). Por otro lado, todos los registros de salinidad fueron mayores de 35 , lo que indicó condiciones hipersalinas (Fig. 2a). Se ha reportado altas salinidades en las lagunas del noroeste de México debido a la alta tasa de evaporación (Álvarez-Borrego y LaraLara, 1991; Páez-Osuna et al., 2016), lo cual está asociado a la profundidad y la tasa de renovación del agua en las lagunas (Bonilla et al., 2005; Pérez-Ruzafa et al., 2005).

La concentración de clorofila a mostró variaciones durante el cultivo experimental con dos picos principales, el mayor en junio $\left(6.5 \mu \mathrm{g} \mathrm{L}^{-1}\right)$ y otro en septiembre $(5.9 \mu \mathrm{g}$ $\left.\mathrm{L}^{-1}\right)$ (Fig. 2b). Este comportamiento es común en lagunas costeras subtropicales y son atribuidos principalmente a los eventos de surgencias marinas (Lara-Rodríguez et al., 2015). La concentración de clorofila a durante el cultivo experimental no parece haber sido influenciada por algún otro parámetro del agua. Por otra parte, el seston presentó dos picos, el mayor en mayo $\left(10.3 \mathrm{mg} \mathrm{L}^{-1}\right)$ con un descenso marcado en junio (5.2 $\left.\mathrm{mg} \mathrm{L}^{-1}\right)$, y luego otro en julio ( $8.9 \mathrm{mg}$ $\mathrm{L}^{-1}$ ) con descenso continuo hasta el final del estudio (Fig. 2b). No se observó correlación del seston con la clorofila, como ha sido reportado por Cervantes-Duarte et al. (2017) en la bahía de La Paz, Baja California Sur, que se encuentra también en el Golfo de California. El seston en la laguna La Cruz podría estar relacionado con los aportes de agua de las granjas camaronícolas y de los asentamientos humanos de Kino Viejo y Kino Nuevo hacia este cuerpo de agua. Lo anterior debido a que el seston se relaciona con aportes antropogénicos (Souchu et al., 2010). En general, las variaciones estacionales, así como los valores de las variables ambientales del agua observados en este estudio coinciden con lo encontrado en reportes anteriores para este cuerpo de agua (Castro-Longoria et al., 1991; Valdez-Holguín, 1994; Gárate-Lizárraga et al., 2013). Con esto se demuestra que las condiciones ambientales no han presentado cambios significativos en los últimos años.

Desde el punto de vista acuícola, las concentraciones de clorofila $a$ y de seston evidenciaron la disponibilidad de alimento para el ostión cultivado. Por ejemplo, se reportó por Lodeiros et al. (2018) un crecimiento óptimo de C. gigas alcanzando la talla comercial en menos de un año bajo concentraciones de seston (2.5-14.5 $\left.\mathrm{mg} \mathrm{L}^{-1}\right)$ similares a las de este estudio.

\section{Crecimiento y condición}

La talla promedio de altura de la concha durante la siembra fue de $4.2 \pm 0.6 \mathrm{~mm}$; posteriormente el crecimiento de los ostiones fue sostenido alcanzando un promedio de $57.7 \pm 7.3 \mathrm{~mm}$ en junio (Fig. 3a). Después el crecimiento 
prácticamente se detiene de julio a octubre, pero se reactiva durante diciembre alcanzando $74.5 \pm 7.95 \mathrm{~mm}$ al final del experimento. Se observó el mismo patrón para la longitud de la concha, iniciando con una talla $1.8 \pm 0.78 \mathrm{~mm}$ para la siembra y posteriormente con crecimiento continuo hasta junio (32.9 $\pm 3.6 \mathrm{~mm}$ ) (Fig. 3a). El crecimiento se mantiene relativamente constante de julio a octubre y se reactiva en diciembre alcanzando $45.3 \pm 5.07 \mathrm{~mm}$ al final del cultivo. La sobrevivencia al término del cultivo experimental fue de 92.3\% y la tasa promedio de crecimiento diaria se estimó en $0.292 \mathrm{~mm} /$ día (altura de concha).

Los resultados indicaron que el crecimiento de los
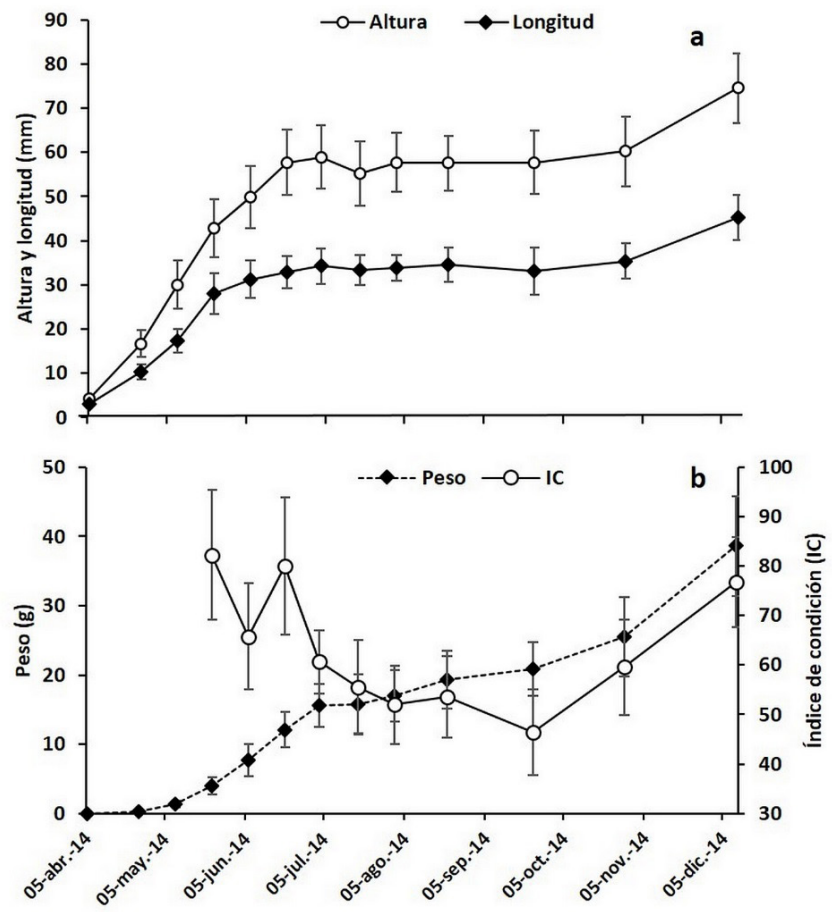

Figura 3. Variación de altura y longitud promedios (a) y de peso e índice de condición promedios (b) de ostiones Crassostrea gigas cultivados en la laguna La Cruz, Sonora.

Figure 3. Mean height and length variation (a), and mean weight and condition index variation (b) of oysters Crassostrea gigas cultivated at La Cruz lagoon, Sonora.

ostiones cultivados estuvo fuertemente influenciado por la temperatura del agua. La relación entre temperatura y crecimiento ha sido reportada para el ostión del Pacífico en México y otros países del mundo (Castillo-Durán et al., 2010; Gamain et al., 2017; Góngora-Gómez et al., 2017; Lodeiros et al., 2018). En otras regiones, las temperaturas mayores a $20^{\circ} \mathrm{C}$ se consideran no óptimas para el desarrollo de C. gigas (Samain et al., 2005). No obstante, en este estudio la temperatura durante la siembra fue de $20^{\circ} \mathrm{C}$ y luego aumentó gradualmente permitiendo un crecimiento sostenido de los organismos hasta junio cuando la temperatura llegó hasta $32.2^{\circ} \mathrm{C}$. Durante ese periodo la clorofila mantuvo valores promedio de $2.4 \pm 0.8 \mu \mathrm{g}$ $\mathrm{L}^{-1}$ y se detectó el pico más importante de seston, sugiriendo que los ostiones tuvieron buena disponibilidad de alimento para soportar el crecimiento. No obstante, aún con abundante alimento disponible (picos de clorofila y seston) de julio a octubre, el efecto de temperaturas mayores a $30^{\circ} \mathrm{C}$ durante este periodo detuvo el crecimiento de los organismos. Este comportamiento coincide con resultados anteriores donde el crecimiento de $C$. gigas se frena significativamente cuando se presentan las temperaturas máximas (Chávez-Villalba et al., 2010). Lo anterior sugiere que en las lagunas del Golfo de California el rango óptimo para el desarrollo de la especie parece estar entre $20-30{ }^{\circ} \mathrm{C}$ debido a décadas de adaptación a las condiciones locales.

El peso promedio de las semillas durante la siembre fue de $0.1 \mathrm{mg}$, luego la ganancia en peso es continua hasta julio alcanzando $15.6 \pm 3.1 \mathrm{~g}$ (Fig. 3b). Posteriormente, la ganancia en peso sigue aumentando, pero a una tasa menor hasta octubre $(25.5 \pm 5.6 \mathrm{~g})$, para incrementar nuevamente $y$ alcanzar $38.6 \pm 7.2 \mathrm{~g}$ al final del estudio. Como se ha mencionado anteriormente, la disponibilidad de alimento durante el periodo de cultivo fue buena, no presentando restricciones para el adecuado desarrollo de los organismos. Por lo tanto, la disminución de ganancia en peso durante verano también se puede atribuir al efecto de las temperaturas más altas. Pero a diferencia de la concha, el peso total de los ostiones no se detiene sino que sólo disminuye, lo que sugiere que lo que sigue aumentando es el peso de la carne. El desacoplamiento entre el crecimiento de la concha y los tejidos ya se había reportado para la especie, pero en la mayoría de los casos ocurre que la concha sigue creciendo mientras que la ganancia en peso se retarda debido a poca disponibilidad de alimento (Brown y Hartwick, 1988). Este patrón también se detectó en ostiones cultivados dentro de la laguna La Cruz cuando se compararon con ostiones cultivados fuera de ésta (Chávez-Villalba et al., 2010). La diferencia observada en este estudio es que, debido a la disponibilidad de alimento, los ostiones canalizaron la energía para formar tejido en lugar de producir concha. Esto también es un indicador de la adaptación de la especie a las condiciones ambientales locales y refleja una ventaja para los ostricultores, ya que los ostiones, aunque dejan de crecer no se "adelgazan" en verano y conservan buenas propiedades para su futura comercialización. Esta estrategia parece surgir después de años de adaptación de los ostiones, ya que aunque la especie tiene su origen natural en aguas templadas, su naturaleza euritérmica y eurihalina (Chávez-Villalba, 2014) le han permitido sobrevivir en las condiciones extremas de las lagunas del Golfo de California. Esta adaptación podría haber favorecido otros procesos como el de fijación de semilla en las lagunas, pero esto tiene que comprobarse en estudios futuros.

El índice de condición (IC) inició con valores altos (82.2 máximo) en mayo y junio para posteriormente disminuir gradualmente y llegar al valor más bajo en septiembre (46.4). Después vuelve a aumentar durante octubre y diciembre hasta alcanzar un valor de 76.8 al final del estudio (Fig. 3b). En general, el IC fue bueno durante todo el experimento debido a que los valores siempre permanecieron por arriba de 50 (excepto septiembre) con un promedio final de $63.2 \pm$ 
12.5. La disminución del IC también correspondió al período de temperaturas altas y reducción de la tasa de ganancia en peso indicando que el verano-otoño es una fase crítica para los ostiones.

El patrón del IC observado aquí es diferente al reportado por Chávez-Villalba et al. (2010) para C. gigas en esta misma laguna, estos autores encontraron valores más bajos, poca variación y no detectaron picos significativos. No obstante, el patrón es similar al descrito por Chávez-Villalba et al. (2007) en la laguna El Soldado, Sonora, también para el ostión del Pacífico, y esos autores no detectaron desoves ni cambios significativos del IC durante la época reproductiva. Se ha descrito que los eventos reproductivos alteran la condición fisiológica de la especie, lo que se ve reflejado en el índice de condición (Suquet et al., 2016). Aquí observamos una disminución significativa del IC durante la época que correspondería a la reproducción de la especie en Bahía Falsa, Baja California (Cáceres-Martínez et al., 2004). No obstante, no tenemos elementos para confirmar que la disminución del IC corresponda a un desove porque no se hicieron análisis histológicos y porque en el momento del evento los ostiones todavía se consideraban juveniles (3-4 meses de edad). Es necesario volver a hacer estudios sobre $C$. gigas en esta laguna para saber si debido a procesos de adaptación, el desarrollo de la reproducción ha cambiado y ahora sea posible que los ostiones desoven y existan fijaciones de semilla. A la fecha, en México no se tienen registros de fijaciones de semilla de la especie en los sitios de cultivo ni en otras áreas (ChávezVillalba, 2014).

\section{Modelo de crecimiento}

En base al criterio de información de Akaike $\left(\mathrm{AIC}_{\mathrm{C}}\right)$, el modelo que mejor describió el crecimiento de $C$. gigas en este estudio fue el Schnute caso 1, con una plausibilidad del $100 \%$ y con la siguiente ecuación:

$$
L_{t}=\left\{4.1^{-0.5}+\left(59.4^{-0.5}-4.1^{-0.5}\right)\left[\frac{1-e^{-20.4(t-0.11)}}{1-e^{-20.4(0.79-0.11)}}\right]\right\}^{\frac{1}{-0.5}}
$$

En general, todos los modelos probados presentaron un buen ajuste a los datos, reflejando la viabilidad de implementar la inferencia multimodelo (IMM) como una alternativa para seleccionar el modelo que mejor describa el crecimiento de la especie. Tradicionalmente, el crecimiento de los bivalvos se modela en base al modelo de von Bertalanffy, sin embargo, se ha cuestionado si realmente es el mejor modelo para este grupo de animales (Cruz-Vázquez et al., 2012). En México se ha modelado el crecimiento de algunos bivalvos usando la IMM, por ejemplo, Góngora-Gómez et al. (2017) compararon los modelos de von Bertalanffy y von Bertalanffy estacional encontrando que el segundo describe mejor el crecimiento de C. gigas. Por otro lado, ChávezVillalba y Aragón-Noriega (2015) compararon seis modelos de crecimiento concluyendo que el modelo Schnute caso 3 es el que mejor modela el crecimiento de $C$. corteziensis. Esto coincide con lo reportado para C. gigas en Argelia, donde se encontró, a través de la IMM, que el modelo Schnute 3 fue el mejor para la especie (Haoui-Meslem et al., 2017). Como se puede apreciar, el crecimiento de la especie y de los bivalvos en general puede ser muy variable dependiendo de las condiciones ambientales donde se desarrollen, por lo que se requiere técnicas como la IMM para encontrar el modelo que mejor describa el crecimiento. No obstante, no se descarta la idea de encontrar un modelo universal para describir el crecimiento de los bivalvos, pero se requieren de más estudios en esta disciplina.

\section{Patógenos}

Los resultados de los análisis de patógenos indicaron que no se registró presencia de OsHV-1, Marteilia refringens y Perkinsus marinus, ni en semilla antes de la siembra ni en adultos al final del cultivo. Estos patógenos han sido reportados en las lagunas del Golfo de California causando mortalidades tanto en los laboratorios de producción como en los cultivos comerciales (Enríquez-Espinoza et al., 2010, 2015; Grijalva-Chon et al., 2013, 2015; De la Re-Vega et al., 2017; Martínez-García et al., 2017). Desde la perspectiva de la sanidad acuícola se puede asumir que los protocolos para prevenir los patógenos en los laboratorios de producción de semilla están dando buenos resultados. Por otra parte, si se considera las variaciones ambientales en el sitio de cultivo y la relación de éstas con la proliferación de patógenos (Solomieu et al., 2015), podemos afirmar que el lote de semillas utilizado en este estudio presenta buena resistencia a enfermedades.

\section{CONCLUSIONES}

La temperatura del agua influyó directamente en el crecimiento y la condición de los ostiones durante su cultivo. Durante los periodos más elevados de temperatura, los ostiones parecen adoptar una estrategia que les permite frenar el crecimiento de la concha y usar la energía proveniente del alimento para formar carne. El estrés que pudiera provocar los cambios extremos de los parámetros del agua no se vio reflejado en infecciones por patógenos a pesar de los múltiples reportes de patógenos asociados a bivalvos en La Cruz. Esto es un indicador de cultivos sanos, lo que contribuye a fortalecer la ostricultura de la región. Desde el punto de vista de la acuacultura, los resultados obtenidos indican un buen desarrollo de los ostiones en cultivo, los cuales están dentro de los estándares de los cultivos comerciales en México. Los modelos de crecimiento fueron sensibles a las variaciones ambientales y su influencia en el crecimiento de los ostiones. Esto indica la importancia de emplear técnicas como la inferencia multimodelo para describir más fielmente el crecimiento de los bivalvos.

\section{AGRADECIMIENTOS}

Se agradece el apoyo técnico de Andrés HernándezIbarra y Josué Valenzuela-Díaz (CIBNOR Unidad Guaymas), Martín Acedo-Valdez (UNISON), Francisco Hoyos-Chairez y 
Víctor Vera-López (CREMES). Este proyecto fue realizado con apoyo de la Fundación Produce Sonora (proyecto 898-1). El primer autor recibió una beca doctoral por parte del CONACYT.

\section{REFERENCIAS}

Álvarez-Borrego, S. y Lara-Lara, J.R. 1991. The physical environment and primary productivity of the Gulf of California. En: The Gulf and Peninsular Province of the Californias. J.P. Dauphin y B. Simoneit (ed.), pp 555-567. American Association of Petroleum Geologist, Tulsa.

Arreola Lizárraga, J.A. 2003. Bases de manejo costero: Patrones ecológicos en la laguna costera Las Guásimas Territorio Yaqui, México. Tesis Doctoral. Centro de investigaciones Biológicas del Noroeste S.C. La Paz B.C.S. México.

Arzul, I., Chollet, B., Boyer, S., Bonnet, D., Gaillard, J., Baldi, Y., Robert, M., Joly, J.P., Garcia, C. y Bouchoucha, M. 2014. Contribution to the understanding of the cycle of the protozoan parasite Marteilia refringens. Parasitology. 141: 227-240.

Audemard, C., Reece, K.S. y Burreson E.M. 2004. Real-time PCR for detection and quantification of the protistan parasite Perkinsus marinus in environmental waters. Applied and Environmental Microbiology. 70: 6611-6618.

Barraza-Guardado, R.H., Chávez-Villalba, J., Atilano-Silva, H. y Hoyoz-Chairez, F. 2009. Seasonal variation in the condition index of Pacific oyster postlarvae (Crassostrea gigas) in a land-based nursery in Sonora, Mexico. Aquaculture Research. 40: 118-128.

Bonilla, S., Conde, D., Aubriot, L. y Pérez, M.C. 2005. Influence of hydrology on phytoplankton species composition and life strategies in a subtropical coastal lagoon periodically connected with the Atlantic Ocean. Estuaries. 28: 884-895.

Brown, J.R. y Hartwick, E.B. 1988. Influences of temperature, salinity and available food upon suspended culture of the Pacific oyster, Crassostrea gigas: I. Absolute and allometric growth. Aquaculture. 70: 231-251.

Burnham, K.P. y Anderson, D.R. 2002. Model selection and multimodel inference: a practical information-theoretic approach. 2th ed. Springer Science \& Business Media. New York.

Cáceres-Martínez, J., Ramírez-Gutiérrez, S., Vazquez-Yeomans, R. y Macías-Montes de Oca, P. 2004, Reproductive cycle and mortality of the Japanese oyster Crassostrea gigas cultured in Bahía Falsa, Baja California, Mexico. Journal of Shellfish Research. 3: 795-801.

Cáceres-Martínez, J., Vásquez-Yeomans, R., Padilla-Lardizábal, G. y Portilla, M.R. 2008. Perkinsus marinus in pleasure oyster Crassostrea corteziensis from Nayarit, Pacific coast of Mexico. Journal of Invertebrate Pathology. 99: 66-73.

Cáceres-Martínez, J., García-Ortega, M., Vásquez-Yeomans, R., Pineda-García, T.J., Stokes, N.A. y Carnegie, R.B. 2012. Natural and cultured populations of the mangrove oyster Saccostrea palmula from Sinaloa, Mexico, infected by Perkinsus marinus. Journal of Invertebrate Pathology. 110: 321-325.

Casas, S.M., La Peyre, J.F., Reece, K.S., Azevedo, C. y Villalba, A. 2002. Continuous in vitro culture of the carpet shell clam Tapes decussatus protozoan parasite Perkinsus atlanticus. Disseases of Aquatic Organisms. 52: 217-231.

Castillo-Durán, A., Chávez-Villalba, J., Arreola-Lizárraga, A. y Barraza-Guardado, R. 2010. Crecimiento comparativo, condición y supervivencia de juveniles de los ostiones
Crassostrea gigas y C. corteziensis cultivados en verano e invierno. Ciencias Marinas. 36: 29-39.

Castro-Longoria, R. y Grijalva-Chon, J.M. 1991. Variabilidad espacio-temporal de nutrientes y seston en la laguna costera La Cruz, Sonora. Ciencias Marinas. 17: 83-97.

Cervantes-Duarte, R., Rodríguez-Mata, L.M. y López-López, S. 2017. Efecto de la marea en la concentración de nutrientes, clorofila $a$ y parámetros físicos y químicos en una laguna costera subtropical (Bahía Magdalena, México). Hidrobiológica. 27: 253:264.

Chávez-Villalba, J., Villelas-Ávila, F. y Cáceres-Martínez, C. 2007. Reproduction, condition and mortality of the Pacific oyster Crassostrea gigas (Thunberg) in Sonora, Mexico. Aquaculture Research. 38: 268-278.

Chávez-Villalba, J., Arreola-Lizárraga, A., Burrola-Sánchez, S. y Hoyos-Chairez, F. 2010. Growth, condition, and survival of the Pacific oyster Crassostrea gigas cultivated within and outside a subtropical lagoon. Aquaculture. 300: 128-136.

Chávez-Villalba, J. 2014. Cultivo de ostión Crassostrea gigas: Análisis de 40 años de actividades en México. Hidrobiológica. 24: 175-190.

Chávez-Villalba, J. y Aragón-Noriega, E. 2015. Modeling the individual growth of the Cortez oyster Crassostrea corteziensis (Bivalvia: Ostreidae) from central Gulf of California. Cahiers de Biologie Marine. 56: 231-236.

Crosby, M.P.y Gale, L.D. 1990. A review and evaluation on bivalve condition index methodologies with a suggested standard method. Journal of Shellfish Research. 9: 233-237.

Cruz-Vásquez, R., Rodríguez-Domínguez, G., Alcántara-Razo, E. y Aragón-Noriega, E.A., 2012. Estimation of individual growth parameters of the Cortes geoduck Panopea globosa from the central Gulf of California using a multimodel approach. Journal of Shellfish Research. 31: 725-732.

De la Re-Vega, E., Sánchez-Paz, A., Gallardo-Ybarra, C., LastraEncinas, M.A., Castro-Longoria, R., Grijalva-Chon, J.M., López-Torres, M.A. y Maldonado-Arce, A.D. 2017. The Pacific oyster (Crassostrea gigas) Hsp70 modulates the Ostreid herpes virus 1 infectivity. Fish and Shellfish Immunology. 71: 127-135.

Diederich, S., Nehls, G., van Beusekom, J.E.E. y Reise, K. 2005. Introduced Pacific oysters (Crassostrea gigas) in the northern Wadden Sea: invasion accelerated by warm summers? Helgoland Marine Research. 59: 97-106.

Enríquez-Espinoza, T., Grijalva-Chon, J., Castro-Longoria, R. y Ramos-Paredes, J. 2010. Perkinsus marinus in Crassostrea gigas in the Gulf of California. Diseases of Aquatic Organisms. 89: 269-273.

Enríquez-Espinoza, T.L., Castro-Longoria, R., Mendoza-Cano, F. y Grijalva-Chon, J.M. 2015. Perkinsus marinus in Crassostrea gigas and Chione fluctifraga from Kino Bay, Sonora, México. Biotecnia, 17: 10-13.

Escobedo-Fregoso, C., Arzul, I., Carrasco, N., Gutiérrez-Rivera, J.N., Llera-Herrera, R. y Vázquez-Juárez, R. 2015. Polymorphism at the ITS and NTS loci of Perkinsus marinus isolated from cultivated oyster Crassostrea corteziensis in Nayarit, Mexico and phylogentic relationship to P. marinus along the Atlantic coast. Transboundary and Emerging Diseases. 62: 137-147.

FAO. 2016. Cultured Aquatic Species Information Program Crassostrea gigas. FAO, Roma. [Consultado 17 de enero 2018]. Disponible en http://www.fao.org/fishery/culturedspecies/ Crassostrea gigas/USA/en. 
FAO 2005-2018. Cultured Aquatic Species Information Programme Crassostrea gigas. Programa de información de especies acuáticas. FAO, Roma. [Consultado 17 enero 2018]. Disponible en: http://www.fao.org/fishery/culturedspecies/ Crassostrea gigas/es.

Fuhrmann, M., Delisle, L., Petton, B., Corporeau, C. y Pernet, F. 2018. Metabolism of the Pacific oyster, Crassostrea gigas, is influenced by salinity and modulates survival to the Ostreid herpesvirus OsHV-1. Biology Open. 7: bio028134.

Gamain, P., Gonzalez, P., Cachot, J., Clérandeau, C., Mazzella, N., Gourves, P.Y. y Morin, B. 2017. Combined effects of temperature and copper and S-metolachlor on embryolarval development of the Pacific oyster, Crassostrea gigas. Marine Pollution Bulletin. 115: 201-210.

Gárate-Lizárraga, I., Poot-Delgado, C.A., Ramírez-Castillo, E.R. y Páez-Hernández,M.H.2013.Proliferación de Pseudo-nitzschia brasiliana y $P$. cf. pseudodelicatissima (Bacillariophyceae) en el Estero Santa Cruz, norte del Golfo de California, México. Revista de Biología Marina y Oceanografía. 48: 393-399.

Gasmi, S., Bernard, I., Pouvreau, S., Maurer, D., Schaal, G., Ganthy, F., Cominassi, L., Allain, G., Sautour B. y David, V. 2017. Spatial patterns in the condition index of the wild Pacific oyster Crassostrea gigas in a macrotidal coastal ecosystem: Influence of tidal processes and beyond. Journal of Sea Research. 119: 28-36.

Gómez-León, A., Rodríguez-Figueroa, G.M., Shumilin, E., Carreño, A.L. y Sánchez, A. 2018. Abundance and distribution of benthic foraminifera as indicators of the quality of the sedimentary environment in a subtropical lagoon, Gulf of California. Marine Pollution Bulletin. 130:31-39.

Góngora-Gómez, A.M., Aragón-Noriega, E.A., DomínguezOrozco, A.L. y Villanueva-Fonseca, B.P. 2017. Modeling the individual growth of the Pacific oyster Crassostrea gigas cultivated in the Gulf of California using the von Bertalanffy model. Revista de Biología Marina y Oceanografía. 52: 181185.

Grijalva-Chon, J.M., Castro-Longoria, R., Ramos-Paredes, J., Enríquez-Espinoza, T.L. y Mendoza-Cano, F. 2013. Detection of a new OsHV-1 DNA strain in the healthy Pacific oyster, Crassostrea gigas, from the Gulf of California. Journal of Fish Diseases. 36: 965-968.

Grijalva-Chon, J.M., Castro-Longoria, R., Enríquez-Espinoza, T.L., Maeda-Martínez, A.N. y Mendoza-Cano, F. 2015. Molecular evidence of the protozoan parasite Marteilia refringens in Crassostrea gigas and Crassostrea corteziensis from the Gulf of California. Latin American Journal of Aquatic Research. 43: 776-780.

Haoui-Meslem, N., Chávez-Villalba, J., Alcántara-Razo, E. y Rebzani-Zahaf, C. 2017. Growth, condition and gametogenic activity of the Pacific oyster Crassostrea gigas in Algeria. Cahiers de Biologie Marine. 58: 153-161.

Katsanevakis, S. 2006. Modelling fish growth: model selection, multi-model inference and model selection uncertainty. Fisheries Research. 81: 229-235.

Lara-Rodríguez, R., Velásquez-Arenas, R., Troccoli-Ghinaglia, L., Hernandez, D. y Lemus, M. 2015. Variación temporal de las variables físico-químicas y biomasa fitoplanctónica en aguas superficiales de la zona costera de Chacopata, NE Venezuela (Mar Caribe). Revista de Biología Marina y Oceanografía. 50: 367-373.

Lodeiros, C., Rodríguez-Pesantes, D., Márquez, A., Revilla, J.,
Chávez-Villalba, J. y Sonnenholzner, S. 2018. Suspended cultivation of the Pacific oyster Crassostrea gigas in the Eastern Tropical Pacific. Aquaculture International. 26: 337347.

López-Flores, I., De La Herrán, R., Garrido-Ramos, M.A., Navas, J.I., Ruíz-Rejón, C. y Ruíz-Rejón, M. 2004. The molecular diagnosis of Marteilia refringens and differentiation between Marteilia strains infecting oysters and mussels based on the rDNA igs sequence. Parasitology. 129: 411-419.

Martínez-García, M.F., Grijalva-Chon, J.M., Castro-Longoria, R., Chávez-Villalba, J.E., Enríquez-Espinoza, T.L., MaedaMartínez, A.N. y Peña-Messina, E. 2017. OsHV-1 and notifiable protozoa in healthy Crassostrea corteziensis cultured in two distant areas of the Gulf of California. Latin American Journal of Aquatic Reseach. 45: 699-707.

Páez-Osuna, F., Sanchez-Cabeza, J., Ruiz-Fernández, A., AlonsoRodríguez, R., Piñón-Gimate, A., Cardoso-Mohedano, J., Flores-Verdugo, F., Carballo, J., Cisneros-Mata, M. y Álvarez-Borrego, S. 2016. Environmental status of the Gulf of California: A review of responses to climate change and climate variability. Earth Science Review. 162: 253-268.

Parsons, T. R., Maitia, Y. y Lalli, C.M. 1984. A Manual of Chemical and Biological Methods for Sea Water Analysis. $1^{\text {st }}$ ed. Pergamonn Press, Oxford.

Pérez-Ruzafa, A., Fernández, A.I., Marcos, C., Gilabert, J., Quispe, J.I. y García-Charton, J.A. 2005. Spatial and temporal variations of hydrological conditions, nutrients and chlorophyll a in a Mediterranean coastal lagoon (Mar Menor, Spain). Hydrobiologia. 550: 11-27.

Renault, T., Le Deuff, Chollet B., Cochennec, N. y Gerard, A. 2000. Concomitant herpes-like virus infections in hatchery-reared larvae and nursery-cultured spat Crassostrea gigas and Ostrea edulis. Diseases of Aquatic Organisms. 42: 173-183.

Rosa-Vélez J., Gutiérrez-Wing, M.T. y Radilla-Camacho, R., 1991. El ostricultivo de Bahía de San Quintín, B.C., México: Aspectos genéticos. Ciencias Marinas. 17: 133-145.

Samain, J.F., Boudry, P., Dégremont, L., Soletchnick, P., Ropert, M., Bédier, E., Martin, J.L., Moal, J., Mathieu, M., Pouvreau, S., Lambert, C., Escoubas, J.M., Nicolas, J.L., Le Roux, F., Renault, T., Burgeot, T. y Bacher, C. 2005. Information on the Distribution, Causes and Significance of the Summer Mortality Syndrome in the Pacific Oyster (Crassostrea gigas) and Other Bivalve Species. Report on the Working Group on Pathology and Diseases of Marine Organisms, ICES CM 2005/F:02, 8-12 March, La Tremblade, France.

Schnute, J. 1981. A versatile growth model with statistically stable parameters. Canadian Journal of Fisheries and Aquatic Sciences. 38: 1128-1140.

Souchu, P., Bec, B., Smith, V. H., Laugier, T., Fiandrino, A., Benau, L., Orsoni, V., Yves, C. y Vaquer, A. 2010. Patterns in nutrient limitation and chlorophyll $a$ along an anthropogenic eutrophication gradient in French Mediterranean coastal lagoons. Canadian Journal of Fisheries and Aquatic Sciences. 67: 743-753.

Strickland, J.D.H. y Parsons, T.R. 1972. A Practical Handbook of Seawater Analysis. Fisheries Research Board of Canada, Bulletin no. 67. Ottawa Canada.

Solomieu, V.B., Renault, T. y Travers, M.A. 2015. Mass mortality in bivalves and the intricate case of the Pacific oyster, Crassostrea gigas. Journal of Invertebrate Pathology. 131: 2-10. 
Suquet, M., Malo, F., Quere, C., Ledu, C., Le Grand, J. y Benabdelmouna, A. 2016. Gamete quality in triploid Pacific oyster (Crassostrea gigas). Aquaculture. 451: 11-15.

Valdez-Holguín, J.E. 1994. Variaciones diarias de temperatura, salinidad, oxígeno disuelto y clorofila a, en una laguna hipersalina del Golfo de California. Ciencias Marinas. 20: 123:137.

Valenzuela-Castillo, A., Sánchez-Paz, A., Castro-Longoria, R., López-Torres, M.A. y Grijalva-Chon, J.M. 2015. Seasonal changes in gene expression and polymorphism of hsp70 in cultivated oysters (Crassostrea gigas) at extreme temperatures. Marine and Environmental Research. 110: 25-32.
Vásquez-Yeomans, R., Cáceres-Martínez, J. y Huerta, A.F. 2004. Herpes-like virus associated with eroded gills of the Pacific oyster Crassostrea gigas in Mexico. Journal of Shellfish Research. 23: 417-419.

Vigneron, V., Solliec, G., Montanié, H. y Renault, T. 2004. Detection of ostreid herpesvirus 1 (OsHv-1) DNA in seawater by PCR: Influence of water parameters in bioassays. Diseases Aquatic Organism. 62: 35-44.

Villanueva-Fonseca, L.C. y Escobedo-Bonilla, C.M. 2013. Prevalencia del protozoario Perkinsus sp. en un cultivo de ostión japonés Crassostrea gigas en Sinaloa, México. Latin American Journal of Aquatic Research. 41: 996-1002. 